\title{
Investigating the Association of IL-1beta IL-8 \& IL 11 with Commonly used Cardiovascular Biomarkers (CK-MB \& Troponin) in Patients with Myocardial Infarction (MI)
}

\section{Hojat Shahbazi}

Fasa University of Medical Science

\section{Mahsa Maleknia}

Fasa University of Medical Science https://orcid.org/0000-0003-0410-132X

Saam Noroozi ( $\nabla$ saam.noroozi@gmail.com )

Fasa University of Medical Science https://orcid.org/0000-0003-2890-6747

\section{Research}

Keywords: myocardial infarction, troponin, CK-MB, IL-1 $\beta$, IL-8, IL-11

Posted Date: May 20th, 2021

DOl: https://doi.org/10.21203/rs.3.rs-524886/v1

License: (c) (1) This work is licensed under a Creative Commons Attribution 4.0 International License.

Read Full License 


\section{Abstract}

Background: Myocardial infarction is a major cause of death and disability worldwide. The term myocardial infarction should be used when there is an evidence of myocardial necrosis in a clinical setting consistent with myocardial ischemia. Cardiac troponins (cTn) have emerged as the preferred diagnostic biomarkers. If troponin assays are not available, the best alternative is creatine kinase (CK$M B$ ). Numerous studies have reported the ability of various inflammatory biomarkers to predict future cardiovascular events. Therefore, we have decided to investigate inflammatory cytokines (IL-1ß, IL-8 \& IL11) to compare with routine biomarkers.

Methods: 40 patients who were diagnosed with myocardial Infarction (at the first 24 hours), have voluntarily participated in this research project. $5 \mathrm{cc}$ of whole blood was collected, both myocardial routine biomarkers (Tropinin \& CK-MB), serum lipid profiles and blood sugar were evaluated after diagnosis of MI. Meanwhile, we have assessed the serum levels of three Interleukins (IL-1 $\beta$, IL-8 \& IL-11) by ELISA method.

Results: First we have assessed blood sugar and all lipid profiles. B.S and HDL were higher in males, while TG, Cholesterol and LDL were higher in females. Our data demonstrated that Troponin continued to increase significantly throughout the 72 hours, while

CK-MB fell dramatically over the period in question. Our result also revealed the rise in IL-1 $\beta$ serum levels (after 72 hours of Ml diagnosis). However, this was not a significant increment. On the other hand, both IL-8 and IL-11 have followed the same decreasing pattern till the end of experiment. It is interesting to note that IL-8 showed a dramatic fall during the experimental period.

\section{Conclusion:}

We concluded that inflammatory cytokines would be a promising diagnostic biomarkers for Ml cases. A noticeable changes of IL-8 plasma levels made it a decent candidate for MI diagnosis and follow-up.

\section{Background}

Myocardial infarction (MI) known as heart attack caused by the deprivation of adequate blood flow in part of the heart muscle which brings about some difficulties such as the lack of oxygen supply and hurting heart muscles. $\mathrm{MI}$ is unfortunately highly associated with death and related difficulties around the world. The main common symptoms are: chest pain, shortness of breath, sweating, nausea, vomiting, abnormal heart beating and some other factors. However, anyone who meets one of the following criteria would be diagnosed for Ml: detecting any changes in cardiac biomarkers (specifically Troponin), ischemia, ECG changes associated with new myocardial ischemia and last but not least is the pathological findings of $\mathrm{MI}(1,2)$. Lots of myocardial infarction cases cannot be diagnosed due to their asymptomatic feature as well as the minor and not specific ones. These are often recognized via the electrocardiogram (ECG) examination that represent the abnormal Q wave. We have to take into account 
that some biomarkers have been released into the blood circulation as a result of heart injury. Researches revealed that these biomarkers might be able to detect ischemia faster and even with higher sensitivity than ECG results. Detectable cardiac biomarkers which are found in the circulation as a consequence of cardiac injury include: myoglobin, cardiac troponin, creatinine kinase (CK), LDH and some other factors. Both troponin and creatinine kinase (specifically CK-MB) have the higher sensitivity and specificity than the other factors mentioned above $(3,4)$.

In terms of sensitivity and tissue-specificity, cardiac troponin is the better match to recognize the myocardial injury rather than some other mentioned biomarkers. Cardiac troponin isoforms (cTnT and cTnl) are measured for myocardial infarction because they can be detected approximately $2-4 \mathrm{~h}$ after the onset of $\mathrm{Ml}$, reach their peak after 24-48 $\mathrm{h}$ and remain in the blood circulation for the next 1-2 days on average $(5,6)$. In times of no availability for troponin assessment, CK-MB could be the great alternative. It depicts the same pattern and kinetics as troponin, with slight differences in details, although it is not comparatively sensitive to detect small MI. The serum levels of CK-MB start to increase $4-9 \mathrm{~h}$ after the beginning of MI symptoms (like troponin), peaks at around $24 \mathrm{~h}$ and finally decreases to reach its baseline within the next 48-72 h. Since it clears from the circulation faster than troponin, it makes this a better biomarker to detect reinfarctions in patients(7-9).

An array of researches depict the association between inflammation and $\mathrm{Ml}$ as well as some other cardiovascular events. Apart from the probable efficiency of routine cardiac biomarkers for diagnosis of myocardial infarction, it has been reported that some inflammation biomarkers would play an important role in either future cardiovascular difficulties' anticipation or diagnosis such as: C-reactive protein (CRP), growth differentiation factor-15 (GDF-15), e interleukin-1 receptor family member called ST2 (ST2), IL-6 and some other interleukins $(10,11)$.

As far as classifying the interleukins concerned, interleukin 1 (IL-1) is the first recognized amongst the other cytokines. IL-1 cDNAs' cloning resulted in two linked isoforms (IL-1a and IL-1 $\beta$ ) with complete different functions. Despite the two isoforms, IL-1 $a$ and IL-1 $\beta$, introduced as IL-1 family, there are 11other members in this family. As for IL-1 $\beta$, unlike IL-1a, a myriad levels of regulation need for its biological activation. Pro-IL-1 $\beta$ is converted into a mature form as a result of proteolytic process caused by IL-1 $\beta$ converting enzyme named caspase-1 $(12,13)$.

Interleukin-8 (IL-8), also known as chemokine C-X-C motif ligand 8 (CXCL8), is typical pro-inflammatory chemokine associated with the recruitment and activation of neutrophil and monocytes. IL-8 gene encodes a protein with 99 amino acids which is finally processed into either a 77 amino acid's protein in nonimmune cells or 72 amino acid's protein in monocytes and macrophages. Although there is an argument over the probable role of IL-8 in ischemia/reperfusion injury, there is still a lack of human researches to confirm this assumption $(14,15)$.

Interleukin-11 (IL-11) is a multifunctional cytokine and member of IL-6 family along with some other cytokines such as: IL-6, IL-31, Cardiotrophin-2 (CT-2), Cardiotrophin-like cytokine (CLC), etc. Although it was discovered just the past quarter century, the minority of investigation has been done on its functions 
and activities. Its multifunctional property recently attracts researchers' attention. There have been some arguments over its effect on various difficulties like inflammations and cancers. There is also the variety of upregulation and downregulation inflammatory responses have been represented as a result of its inflammatory microenvironment. Studies showed that IL-11 might take part in cardiovascular diseases via targeting cardio myocytes in order to reduce the cardiovascular injuries $(16,17)$.

In spite of several studies on the effectiveness of main cardiac biomarkers (Troponin and CK-MB) on MI prognosis and follow up, there are still some doubt over their complete sensitivity and specificity. Thus, the present study was designed to recognize the probable role of inflammatory biomarkers (IL-1 $\beta$ - IL-8 IL-11) to facilitate the MI diagnosis and also their follow up advantages. We assume that the simultaneous measurements of both routine biomarkers and inflammatory ones would make it easier to diagnose $\mathrm{Ml}$ as well as the probability of reinfarction in these patients.

\section{Material And Methods}

\section{Study population}

40 patients, who were diagnosed with Myocardial Infarction (at the first 24 hours of stroke), have participated voluntarily in this research project. We have to take into account that all the patients with the history of inflammation and cancer diseases or whoever diagnosed with any type of diseases resulted in increment in the amount of inflammatory factors, have been omitted from the project. All participants were thoroughly informed prior to the study and also before taking part in the research. Their informed consents were also obtained before sampling. This study was approved and carried out by following the ethical guidelines and regulations which were confirmed by ethics committee of the Fasa University of Medical Sciences.

\section{Blood Sample Collection and Interleukin measurements}

$5 \mathrm{cc}$ of whole blood were collected by seasoned nurses. Then all blood samples were centrifuged to obtain their serum. Both routine myocardial biomarkers (Tropinin \& CK-MB) were evaluated as the first steps of MI diagnosis. All serum lipid profiles and their blood sugar were evaluated as well. Subsequently, we have assessed the serum levels of three Interleukins (IL-1 $\beta$, IL-8 \& IL-11) using the enzyme-linked immunosorbent assay (ELISA) kits following the manufacturer's instructions (Bioassay Technology Laboratory- China).

\section{Follow-up}

After the first round of sampling we would like to follow up the patient's condition, via the measurements of both biomarkers and mentioned interleukins, 72 hours after the onset of MI. Therefore, the same amount of blood samples has been collected for the assessment of three interleukins' (IL-1 $\beta$ - IL-8 - IL- 
11) serum levels as well as the main biomarkers (Troponin \& CK-MB), in order to follow-up the patients' conditions. This could make it easier to compare the probable effects of cardiac biomarkers and inflammatory biomarkers for the MI cases follow-up.

\section{Statistical analyses}

All the data was represented as mean \pm standard error (SEM). SPSS statistical software (SPSS, Chicago, IL, USA, version 21) was applied to analyze the data. The Wilcoxon test was used to compare data obtained from the variables (Troponin, CK-MB, IL-13, IL-8 and IL-11). Spearman's correlation coefficient was employed to establish the relationship between changes in the values of parameters. Continuous variables were compared using the Wilcoxon signed-rank test. Normal distribution of data was checked using the Shapiro-Wilk test $(P<0.05) . P \leq 0.05$ was considered to be statistically significant.

\section{Results}

In this study we have investigated cardiac biomarkers (troponin and CK-MB) in $40 \mathrm{Ml}$ patients. We also have evaluated three different interleukins in order to compare with routine biomarkers. First their blood sugar and lipid profiles were measured in both sexes. It is interesting to note that B.S and HDL were higher in males, while TG, Cholesterol and LDL were higher in females (Table 1).

Table 1

The evaluation of Blood Sugar and Lipid Profiles in both sexes

\begin{tabular}{|lll|}
\hline Biochemical Factors & Male & Female \\
\hline B.S & $180.08 \pm 28.034$ & $159.71 \pm 16.443$ \\
\hline TG & $167.67 \pm 13.809$ & $175.25 \pm 14.332$ \\
\hline Cholesterol & $145.00 \pm 12.485$ & $157.00 \pm 9.366$ \\
\hline HDL & $34.00 \pm 2.634$ & $32.54 \pm 2.041$ \\
\hline LDL & $79.00 \pm 9.489$ & $92.86 \pm 7.149$ \\
\hline
\end{tabular}

\section{Troponin \& CK-MB}

Our data demonstrated that Troponin started its increment from the first sampling after MI verification. Then it has been reached its peak after 72 hours of diagnosis to around $200 \mathrm{ng} / \mathrm{ml}$. CK-MB followed a complete different pattern. Following the onset of myocardial infarction symptoms, it increased in serum dramatically (at first 3-6 hours). However, the amounts have been decreased significantly 72 hours after Ml diagnosis (Figs. 1 \& 2). 
1st time point: showed the first serum troponin measurements after the MI verification. 2 nd time point: showed the second serum troponin measurements 72 hours after Ml verification. Results are presented as mean $\pm S E M, n=40$. $P \leq 0.05^{*}$.

1st time point: showed the first serum troponin measurements after the MI verification. 2 nd time point: showed the second serum troponin measurements 72 hours after MI verification. Results are presented as mean $\pm S E M, n=40$. $P \leq 0.05^{*}$.

\section{Inflammatory Biomarkers}

Our result revealed the increasing pattern for IL-1 $\beta$ over the time past. However, the measurements showed no significant rise within the second sampling time (time point 2) compared with the time 1 (Fig. 3). On the other hand, both IL-8 and IL-11 have followed the same pattern of decreasing throughout the experimental time, which means they both decreased after 72 hours. It is interesting to note that just IL-8 illustrated a dramatic decrement during the whole experimental time (Fig. 4,5).

1st time point: showed the first serum troponin measurements after the MI verification. 2nd time point: showed the second serum troponin measurements 72 hours after MI verification. Results are presented as mean $\pm S E M, n=40$. $P \leq 0.05^{*}$.

1st time point: showed the first serum troponin measurements after the MI verification. 2nd time point: showed the second serum troponin measurements 72 hours after MI verification. Results are presented as mean $\pm S E M, n=40$. $P \leq 0.05^{\star}$.

1st time point: showed the first serum troponin measurements after the MI verification. 2nd time point: showed the second serum troponin measurements 72 hours after of MI verification. Results are presented as mean $\pm S E M, n=40$. $P \leq 0.05^{*}$.

\section{Discussion}

In the present study we investigate three interleukins (IL-1 $\beta$ - IL-8 and IL-11) as probable and practical biomarkers along with cardiac biomarkers (troponin and CK-MB) in order to have efficient and timely diagnosis of MI. Since the sensitivity and specificity of biomarkers for diagnosing myocardial injuries is of high importance, we could take the advantage of inflammatory biomarkers assessments as a complementary way of diagnosis as well as follow-up procedure, together with the routine biomarkers. These would be beneficial to follow-up patients in terms of treatment steps and anticipating eventual reinfarction.

In the current research, blood sugar and lipid profiles of each patient (both sexes) were measured at the beginning of the experiment. A higher amounts of blood sugar and HDL have been reported in males, while TG, cholesterol and LDL were higher in females. Haseeb A. Khan et al noticed that total cholesterol (TC), LDL and HDL decreased significantly in acute myocardial infarction patients (AMI). They mentioned 
that although TC reduction did not prevent the AMI risk, a decrement in HDL would make these patients vulnerable to AMI incidence(18). Also, Amit Kumar Shrivastava and his colleagues, who evaluated 400 AMI patients, illustrated a significant decrease in TC, LDL and HDL and a remarkable elevation in TG and inflammatory markers such as : IL- 6 and IL-10 within the first 2 days of sampling. They represented the serum level's correction of mentioned factors at 7 days of AMI onset. Thus, the assessments of serum lipid profiles are highly recommended after the MI diagnosis in order to reduce the risks(19). There are also more researches which highlights the importance of lipids and lipoprotein measurements in cardiovascular diseases like Bahattin Balci who underlined An increase in TG and low density lipoproteins and decrease in LDL, HDL and TC in patients with Acute Coronary Syndrome (ACS). He suggested that fasting lipid profile assessment and statin therapy for these patients, as long as they admit to the hospital, would probably reduce morbidity and mortality(20).

Studies have claimed that both gender and age differences have influenced the amounts of serum lipid profiles in patients with MI. In 2017 Zhixiong Zhong et al showed the higher TC, LDL, HDL and TG levels in females than males in elderly Hakka patients with acute myocardial infarction in southern China. They also mentioned a higher chance of dyslipidemia in non-elderly male patients than elderly one. Therefore, they had a great suggestion of lipid-lowering therapy for their elderly patients in order to decrease the cardiovascular difficulties' risks(21). Our study confirms that the amount of serum lipid profile is sexdependent and any contradiction between these studies would stem from a population differences.

In some recent studies many researches have introduced how serum lipid profile evaluation could be essential within the 24 hours of admission with acute coronary syndrome symptoms. In 2018 William T. Wang et al depicted that post-MI follow-up of patients in terms of their serum lipid profiles were taken for granted throughout the clinical processes, although the American College of Cardiology/American Heart Association guidelines emphasized on the positive influence of lipid-lowering therapy immediately after the patient's admission and discharge. In spite of a periodically decreasing pattern for TC, LDL, and HDL to reach their plateau in MI patients, lipid-lowering therapy should be begun in the first days of diagnosis. In addition, in 2019 Naresh Kumar et al reported the serum lipid profile changes in the first 24 hours of and after 48 hours of MI incident. The decrement of TC, LDL and HDL and the increment of TG showed over a period in question. All in all, the phasic evaluation of lipid profiles would probably make it easier to follow their trend, making a wise decision toward the application of lipid-lowering therapy for MI patients in no time $(22,23)$.

In the current study the amount of Troponin and CK-MB were changed with the same pattern similar to the finding of other researches. TnC blood levels increased within the first hours of patient's admission, continuing its rise to reach its peak and stay high for the next 2-3 weeks. Although CK-MB demonstrated the same increasing trend as $\mathrm{TnC}$, it has decreased to reach its normal levels with the next 48-72 hours. Various researches depicted that CK-MB is no longer evaluated as an emergency biomarker and could be used to estimate infarct size and prognosis processes $(24,25)$. 
Due to the importance of having biomarkers with high sensitivity and specificity for MI diagnosis along with patient's follow-up, we decided to investigate some inflammatory cytokines (1L-1ß, IL-8 \& IL-11) and compare them with the routine biomarkers.

Regarding the IL-1 $\beta$ blood levels, the increasing pattern has been depicted not only in the first sampling but after 72 hours. This showed a marked similarity with the troponin changes throughout the experimental period. In a recent study Johanne Silvain et al. found that high IL-1 $\beta$ levels were closely associated with cardiovascular mortality, major cardiovascular events (MACE) and as a whole all-cause mortality. These findings were represented as a result of 90-days and one-year follow up of patients with acute MI. They have also support the necessity of using IL-1 $\beta$ inhibitors in the acute MI patients as a treatment and risk reductions(26). Paul M Ridker et al. reported that the anti-inflammatory therapy approach toward IL-1 $\beta$ with Canakinumab, a therapeutic monoclonal antibody targeting interleukin-1 $\beta$, without any changes of LDL cholesterol levels could substantially reduce the cardiovascular diseases' incidents in the patients with the MI history(27). According to the results of another study, it has been demonstrated that IL-1 $\beta$ levels were in connection with repairing process of patients with the MI history(STEMI). They indeed mentioned the importance of this inflammatory cytokine and dysfunctional myocardial remodeling after the reperfused $\mathrm{Ml}$ that would bring about myocardial function impairment and heart failure(28).

In the recent letter published in 2019 Hirotaka Mor et al. illustrated that that nolotinib, prescribing for chronic myeloid leukemia (CML) patients, could increase the IL-1 $\beta$ serum levels and cardiovascular events. Despite the effectiveness of nilotinib for CML, it should not be prescribed for patients with the cardiovascular history and risk factors. They have also showed that IL-1 $\beta$ might contribute to apoptosis through releasing from injured cardio myocytes in the AMI patients. Apoptosis was induced by IL-1 $\beta$ in cardio myocytes of murine MI models. Taken together, IL-1 $\beta$ would elevate the risk of apoptosis and should be controlled specifically in high risk patients(29). Recently Mona Panahi et al. were represent the adverse effects of post-MI inflammation which led to heart failure (HF). A timely decision like blockade of inflammatory cytokines (IL-1) could prevent the incidence of post-MI excessive inflammation which curb the repairing processes by immune system(30).

Moreover, myriad of researches have demonstrated the plausible association between IL-1 $\beta+3954 \mathrm{C} / \mathrm{T}$ polymorphism (rs1143634) and $\mathrm{Ml}$ incidence. This has been proved via a meta-analysis by Yizhen Fang et al., specifically amongst Caucasian populations(31). We have to take into account that although the circulation levels of IL-1 $\beta$ is very low to investigate, this inflammatory biomarkers' assessment would be beneficial in order to eliminate its probable adverse impact on cardiovascular remodeling in reperfused $\mathrm{MI}$ patients.

We also studied IL-8 and IL-11 in MI cases. Both inflammatory biomarkers followed the same falling pattern in an experimental period. IL-8 has decreased dramatically after the 2nd time point, while IL-11 changes was not statistically significant. Kathrin Schömig et al. showed the association between IL-8 levels and progenitor cells in circulation of AMI patients. Progenitor cells are released during 
inflammation, giving rise to neovascularization. This would clarify the possibility of beneficial impacts induced by inflammatory biomarkers instead of their mere adverse effects(32). On the contrary Christian Shetelig et al. (2018) reported the link between IL-8 high levels and some cardiovascular difficulties in STEMI patients such as: microvascular obstruction, large infarct size, LV remodeling and further clinical post-MI incidences. They have supposed the possible IL-8's therapeutic approach toward identifying high risk STEMI patients as well as post- infarct inflammation reduction(14). Our result confirms the increase in the IL-8 serum levels with the onset of MI. Owing to the probable effects of this inflammatory biomarker to deteriorate the post-MI conditions, it could have been used as an inflammatory regulation target in post-infarct patients in order to curb new events.

In another recent review in 2018 researches have provided the results related to the benefits of cytokines' (IL-1, -6, -8, MCP-1, CC chemokines, CXC chemokines and TNF-a) inhibition on cardiac function. However, adverse or even neutral results was indeed represented for some of these cytokines (IL-1, IL-6, IL-8, and MCP-1). There were still some conflicting animal model results. They have showed the use of monoclonal antibody against IL-8 for ischemic reperfusion rabbit model and its association with infarct size reduction, while in another study the IL-8 receptor overexpression in a chronic $\mathrm{MI}$ rat model caused a decrease in infarct size and inflammatory cells and finally LEVF improvement(33). In a case-control study by S Zarrouk-Mahjoub et al., $30 \mathrm{Ml}$ patients have been compared with 30 healthy individuals (controls) in terms of their serum concentrations of interleukin-8 (IL8), tumor-necrosis factor-alpha (TNFa) and interleukin-10 (IL10), pro- and anti-inflammatory cytokines, on days 2 and 30 of post-MI. They illustrated the rise in pro-inflammatory cytokines (IL-8 and TNF-a) along with the fall in anti-inflammatory one, IL-10. This inequality between pro and anti-inflammatory cytokines would lead to heart failure(34). Our results reconcile with the previous studies regarding the increased IL-8 levels just after MI onset.

Since there was a belief about the link between inflammation and coronary heart disease (CHD), Zhengxia Liu et al. have evaluated the inflammatory cytokines levels (CD121a, interleukin [IL]-1ß, IL-8, and IL-11) in CHD and CHD-free patients. Although the CD121a levels were high in MI patients, IL-1 $\beta$ levels were unchanged throughout the experiment. Like CD121a, IL-8 levels were also high in AMI patients. Meanwhile they have mentioned the highest levels of IL-11 in CHD patients rather than non-CHD ones(35). In a case report in 2016, 4 different cases have been treated with IL-11, known as a cardio protective cytokine, in ST elevation myocardial infarction (STEMI) patients. There was not any adverse drug reaction throughout the treatment with recombinant human IL-11 (rhIL-1) and ultimately all cases left the hospital without any HF symptoms(36).

According to the IL-11 treatment, Masanori Obana et al. have illustrated that IL-11 treatment for coronary artery ligation animal model could alleviate post MI cardiac fibrosis via STAT3. They have proved a notable upregulation of IL-11 mRNA in cardiac myocytes of MI cases. Therefore, intravenous administration of IL-11, almost after ligation, could decrease the fibrosis area. These all gave a new perspective for IL-11 treatment against post-MI complications(37). In a recent study in 2019 researchers have investigated the association between IL-11 levels and cardiac prognosis of chronic HF patients. Although the specific mechanisms of IL-11 remained unknown, higher levels of IL-11 have been reported 
throughout the cardiac events which might have severe outcomes(38). Our result confirmed the IL-11 increment that has been reported throughout the cardiac evets in mentioned studies. Moreover, due to the conflicting evidences from different researches we suggest a thorough evaluation of pro and antiinflammatory cytokines as well as a long term follow-up till the patients' stable conditions.

Some limitations can be enumerated for present study. The sample size was relatively small owing to the lack of enough volunteers to join the research study. Furthermore, we recommend the evaluation of various related pro and anti-inflammatory cytokines simultaneously along with in depth clinical $\mathrm{MI}$ and $\mathrm{HF}$ investigations in the hospital to conduct a concrete conclusion.

\section{Conclusion}

In conclusion, lipid profile variations should be taken into consideration as crucial factors which curb the incidence of reinfarction and cardiac deterioration. Moreover, inflammatory cytokines, both proinflammatory and anti-inflammatory ones, would be beneficial to apply as routine biomarkers for MI diagnosis and follow-up. Significant changes in IL-8 would show the importance of this cytokine as a diagnostic factor within the onset of the MI.

\section{Declarations}

\section{Availability of data and materials}

The datasets used and/or analyzed during the current study are available from the corresponding author on reasonable request.

\section{Funds}

This research did not receive any specific grant from funding agencies in the public, commercial, or notfor-profit sectors for the research, authorship, and/or publication of this article.

\section{Acknowledgments}

The authors would like to acknowledge the Fasa University of Medical Sciences for providing facilities and laboratory equipment for this study.

\section{Declaration of interest}

The authors declare no conflicts of interest. 


\section{Author contribution}

Dr. Saam Noroozi and Mahsa Maleknia have conceived and designed the research. Hojat Shahbazi and Mahsa Maleknia have conducted the experiments as well as collecting and analyzing the data. Dr. Saam Noorzi and Mahsa Maleknia have written the original draft. All the authors read and approved the manuscript and all data were generated in-house.

\section{References}

1. Lu L, Liu M, Sun R, Zheng Y, Zhang P. Myocardial Infarction: Symptoms and Treatments. Cell biochemistry and biophysics. 2015;72(3):865-7.

2. Thygesen K, Alpert JS, White HD. Universal definition of myocardial infarction. Journal of the American College of Cardiology. 2007;50(22):2173-95.

3. Howie-Esquivel J, White M. Biomarkers in acute cardiovascular disease. The Journal of cardiovascular nursing. 2008;23(2):124-31.

4. Valensi P, Lorgis L, Cottin Y. Prevalence, incidence, predictive factors and prognosis of silent myocardial infarction: a review of the literature. Archives of cardiovascular diseases. 2011;104(3):178-88.

5. Fathil MFM, Md Arshad MK, Gopinath SCB, Hashim U, Adzhri R, Ayub RM, et al. Diagnostics on acute myocardial infarction: Cardiac troponin biomarkers. Biosensors and Bioelectronics. 2015;70:209-20.

6. Morrow DA, Cannon CP, Jesse RL, Newby LK, Ravkilde J, Storrow AB, et al. National Academy of Clinical Biochemistry Laboratory Medicine Practice Guidelines: Clinical characteristics and utilization of biochemical markers in acute coronary syndromes. Circulation. 2007;115(13):e356-75.

7. Mythili S, Malathi N. Diagnostic markers of acute myocardial infarction. Biomed Rep. 2015;3(6):7438.

8. Reed GW, Rossi JE, Cannon CP. Acute myocardial infarction. Lancet (London, England). 2017;389(10065):197-210.

9. Thygesen K, Alpert JS, Jaffe AS, Chaitman BR, Bax JJ, Morrow DA, et al. Fourth Universal Definition of Myocardial Infarction (2018). Journal of the American College of Cardiology. 2018;72(18):223164.

10. Minamisawa M, Motoki H, Izawa A, Kashima Y, Hioki H, Abe N, et al. Comparison of Inflammatory Biomarkers in Outpatients With Prior Myocardial Infarction. International Heart Journal. 2016;advpub.

11. Moriya J. Critical roles of inflammation in atherosclerosis. Journal of cardiology. 2019;73(1):22-7.

12. Dinarello CA. Interleukin-1 in the pathogenesis and treatment of inflammatory diseases. Blood. 2011;117(14):3720-32.

13. Libby P. Interleukin-1 Beta as a Target for Atherosclerosis Therapy: Biological Basis of CANTOS and Beyond. Journal of the American College of Cardiology. 2017;70(18):2278-89. 
14. Shetelig C, Limalanathan S, Hoffmann P, Seljeflot I, Gran JM, Eritsland J, et al. Association of IL-8 With Infarct Size and Clinical Outcomes in Patients With STEMI. Journal of the American College of Cardiology. 2018;72(2):187-98.

15. Waugh DJJ, Wilson C. The Interleukin-8 Pathway in Cancer. Clinical Cancer Research. 2008;14(21):6735.

16. Kimura R, Maeda M, Arita A, Oshima $Y$, Obana $M$, Ito $T$, et al. Identification of cardiac myocytes as the target of interleukin 11, a cardioprotective cytokine. Cytokine. 2007;38(2):107-15.

17. Negahdaripour M, Nezafat N, Ghasemi Y. A panoramic review and in silico analysis of IL-11 structure and function. Cytokine \& growth factor reviews. 2016;32:41-61.

18. Khan HA, Alhomida AS, Sobki SH. Lipid profile of patients with acute myocardial infarction and its correlation with systemic inflammation. Biomark Insights. 2013;8:1-7.

19. Shrivastava AK, Singh HV, Raizada A, Singh SK. Serial measurement of lipid profile and inflammatory markers in patients with acute myocardial infarction. EXCLI J. 2015;14:517-26.

20. Balci B. The modification of serum lipids after acute coronary syndrome and importance in clinical practice. Curr Cardiol Rev. 2011;7(4):272-6.

21. Zhong Z, Liu J, Li B, Li C, Liu Z, Yang M, et al. Serum lipid profiles in patients with acute myocardial infarction in Hakka population in southern China. Lipids Health Dis. 2017;16(1):246-.

22. Kumar N, Kumar S, Kumar A, Shakoor T, Rizwan A. Lipid Profile of Patients with Acute Myocardial Infarction (AMI). Cureus. 2019;11(3):e4265-e.

23. Wang WT, Hellkamp A, Doll JA, Thomas L, Navar AM, Fonarow GC, et al. Lipid Testing and Statin Dosing After Acute Myocardial Infarction. J Am Heart Assoc. 2018;7(3):e006460.

24. Aydin S, Ugur K, Aydin S, Sahin I, Yardim M. Biomarkers in acute myocardial infarction: current perspectives. Vasc Health Risk Manag. 2019;15:1-10.

25. Suleiman HM, Aliyu IS, Abubakar SA, Isa MS, El-Bashir JM, Adamu R, et al. Cardiac Troponin T and creatine kinase $M B$ fraction levels among patients with acute ischemic stroke in Nigeria. Nigerian journal of clinical practice. 2017;20(12):1618-21.

26. Silvain J, Kerneis M, Zeitouni M, Lattuca B, Galier S, Brugier D, et al. Interleukin-1 $\beta$ and Risk of Premature Death in Patients With Myocardial Infarction. Journal of the American College of Cardiology. 2020;76(15):1763-73.

27. Ridker PM, Everett BM, Thuren T, MacFadyen JG, Chang WH, Ballantyne C, et al. Antiinflammatory Therapy with Canakinumab for Atherosclerotic Disease. New England Journal of Medicine. 2017;377(12):1119-31.

28. Ørn S, Ueland T, Manhenke C, Sandanger $\varnothing$, Godang K, Yndestad A, et al. Increased interleukin-1 $\beta$ levels are associated with left ventricular hypertrophy and remodelling following acute ST segment elevation myocardial infarction treated by primary percutaneous coronary intervention. Journal of internal medicine. 2012;272(3):267-76. 
29. Mori H, Sukegawa M, Fukatsu M, Sano T, Takahashi H, Harada K, et al. The link between interleukin$1 \beta$ and acute myocardial infarction in chronic myeloid leukemia patients treated with nilotinib: crosssectional study. Annals of hematology. 2020;99(2):359-61.

30. Panahi M, Papanikolaou A, Torabi A, Zhang J-G, Khan H, Vazir A, et al. Immunomodulatory interventions in myocardial infarction and heart failure: a systematic review of clinical trials and meta-analysis of IL-1 inhibition. Cardiovasc Res. 2018;114(11):1445-61.

31. Fang Y, Xie H, Lin Z. Association between IL-1 $\beta+3954 C / T$ polymorphism and myocardial infarction risk: A meta-analysis. Medicine (Baltimore). 2018;97(30):e11645-e.

32. Schömig K, Busch G, Steppich B, Sepp D, Kaufmann J, Stein A, et al. Interleukin-8 is associated with circulating CD133+ progenitor cells in acute myocardial infarction. European heart journal. 2006;27(9):1032-7.

33. Hartman MHT, Groot HE, Leach IM, Karper JC, van der Harst P. Translational overview of cytokine inhibition in acute myocardial infarction and chronic heart failure. Trends in cardiovascular medicine. 2018;28(6):369-79.

34. Zarrouk-Mahjoub S, Zaghdoudi M, Amira Z, Chebi H, Khabouchi N, Finsterer J, et al. Pro- and antiinflammatory cytokines in post-infarction left ventricular remodeling. International journal of cardiology. 2016;221:632-6.

35. Liu Z, Zhang M, Wu J, Zhou P, Liu Y, Wu Y, et al. Serum CD121a (Interleukin 1 Receptor, Type I): A Potential Novel Inflammatory Marker for Coronary Heart Disease. PLoS One. 2015;10(6):e0131086-e.

36. Nakagawa M, Owada Y, Izumi Y, Nonin S, Sugioka K, Nakatani D, et al. Four cases of investigational therapy with interleukin-11 against acute myocardial infarction. Heart and vessels. 2016;31(9):15748.

37. Obana M, Maeda M, Takeda K, Hayama A, Mohri T, Yamashita T, et al. Therapeutic Activation of Signal Transducer and Activator of Transcription 3 by Interleukin-11 Ameliorates Cardiac Fibrosis After Myocardial Infarction. Circulation. 2010;121(5):684-91.

38. Ye J, Wang Z, Ye D, Wang Y, Wang M, Ji Q, et al. Increased Interleukin-11 Levels Are Correlated with Cardiac Events in Patients with Chronic Heart Failure. Mediators of Inflammation. 2019;2019:1575410.

\section{Figures}




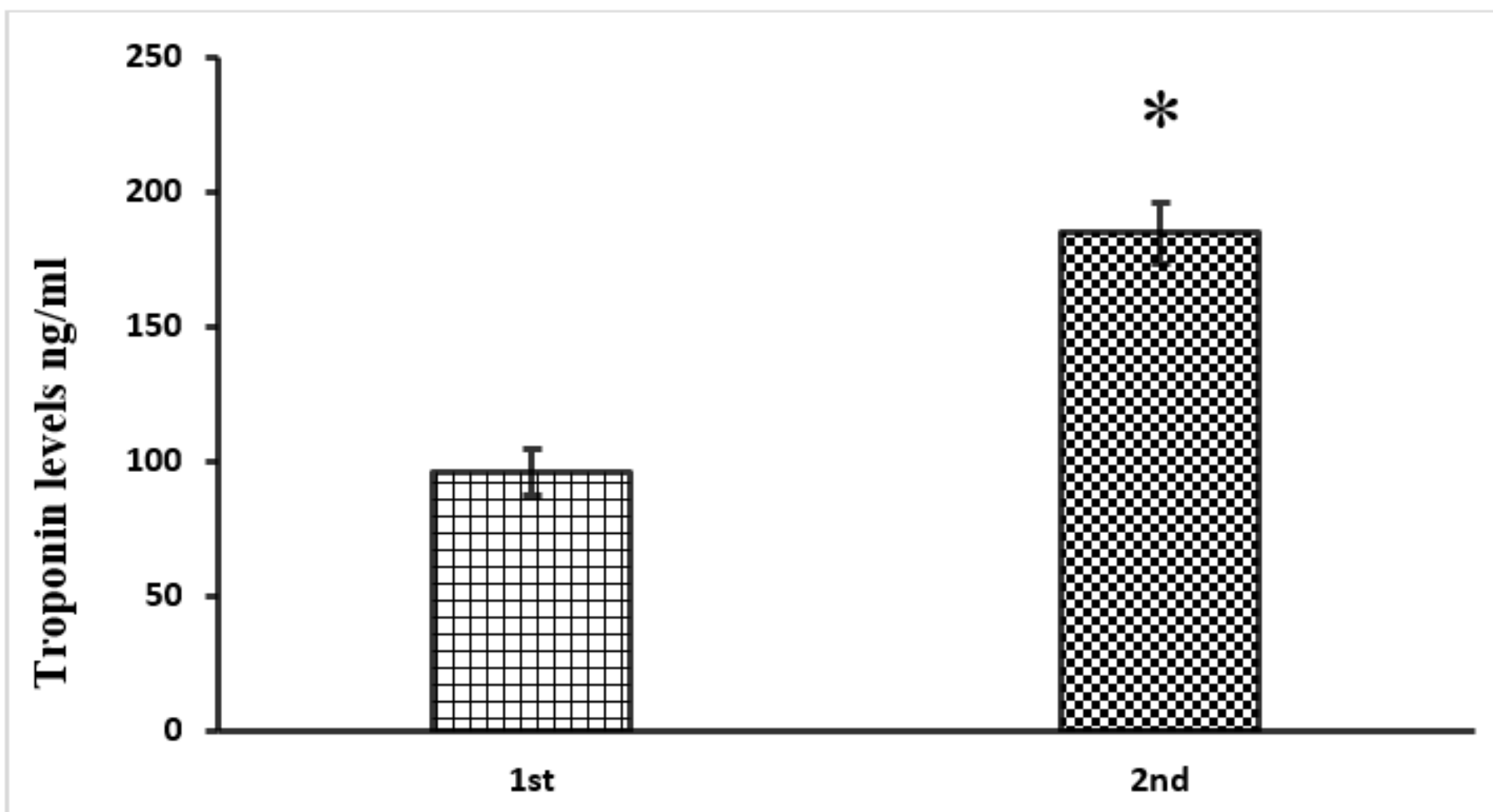

Time

\section{Figure 1}

Serum troponin $(\mathrm{ng} / \mathrm{ml})$ concentrations in patients with myocardial infarction. 1st time point: showed the first serum troponin measurements after the MI verification. 2nd time point: showed the second serum troponin measurements 72 hours after Ml verification. Results are presented as mean $\pm S E M, n=40$. $\mathrm{P} \leq 0.05^{\star}$. 


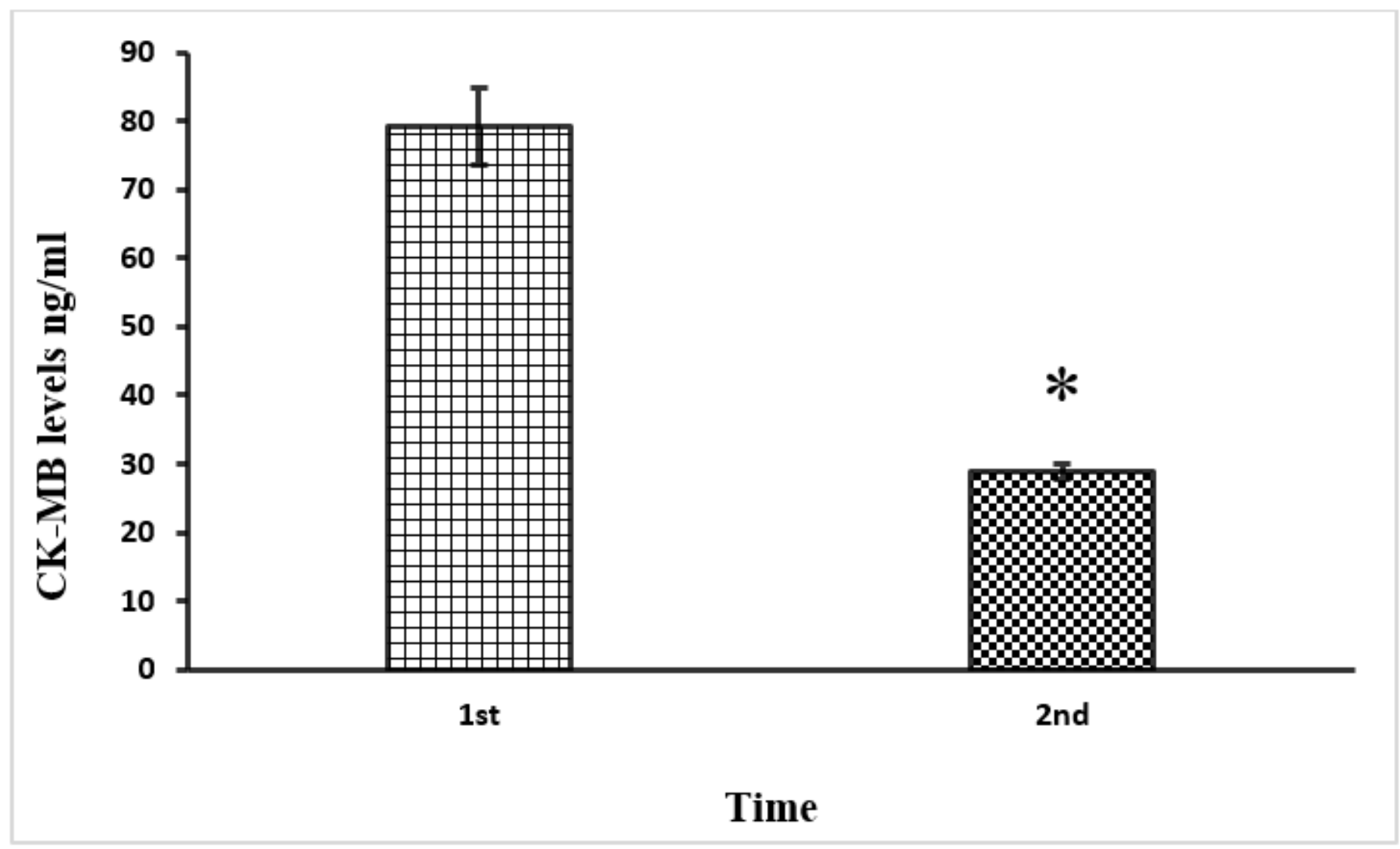

Figure 2

Serum CK-MB $(\mathrm{ng} / \mathrm{ml})$ concentrations in patients with myocardial infarction. 1st time point: showed the first serum troponin measurements after the MI verification. 2nd time point: showed the second serum troponin measurements 72 hours after MI verification. Results are presented as mean $\pm S E M, n=40$. $\mathrm{P} \leq 0.05^{\star}$. 


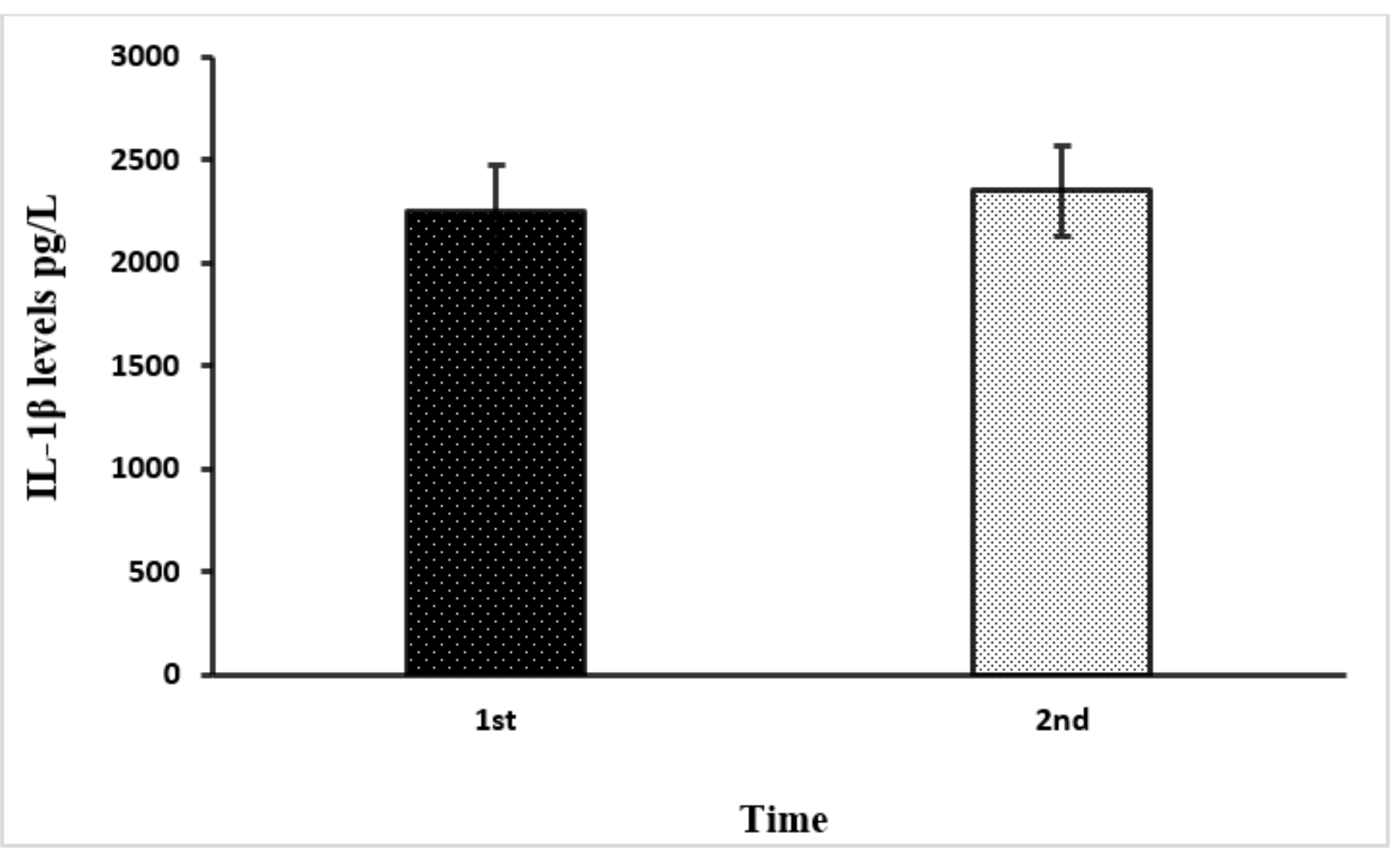

Figure 3

Serum IL-1 $\beta$ (pg/L) concentrations in patients with myocardial infarction. 1st time point: showed the first serum troponin measurements after the MI verification. 2nd time point: showed the second serum troponin measurements 72 hours after MI verification. Results are presented as mean $\pm S E M, n=40$. $\mathrm{P} \leq 0.05^{\star}$. 


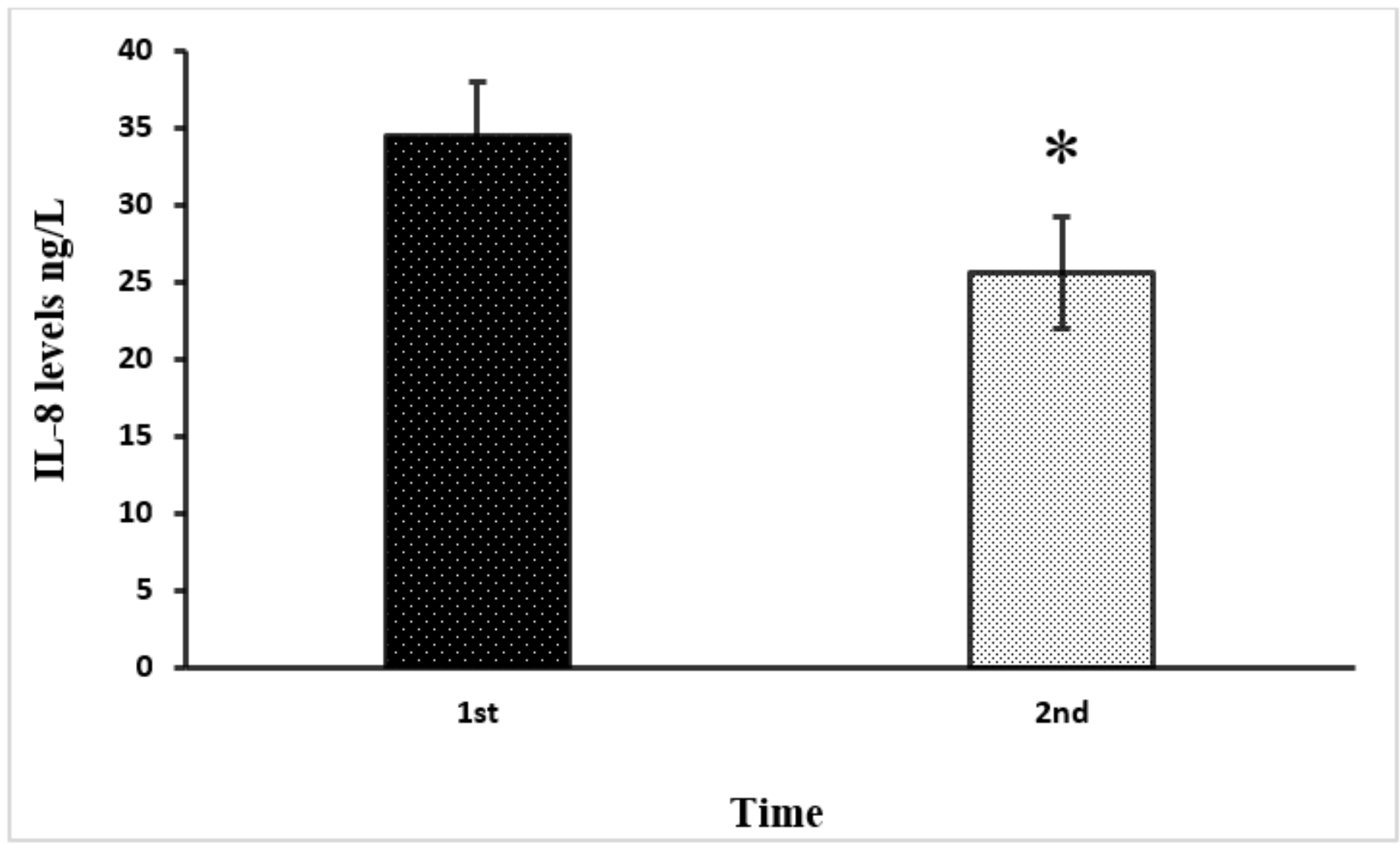

Figure 4

Serum IL-8 ( $\mathrm{ng} / \mathrm{L})$ concentrations in patients with myocardial infarction. 1st time point: showed the first serum troponin measurements after the MI verification. 2nd time point: showed the second serum troponin measurements 72 hours after MI verification. Results are presented as mean $\pm S E M, n=40$. $\mathrm{P} \leq 0.05^{\star}$. 


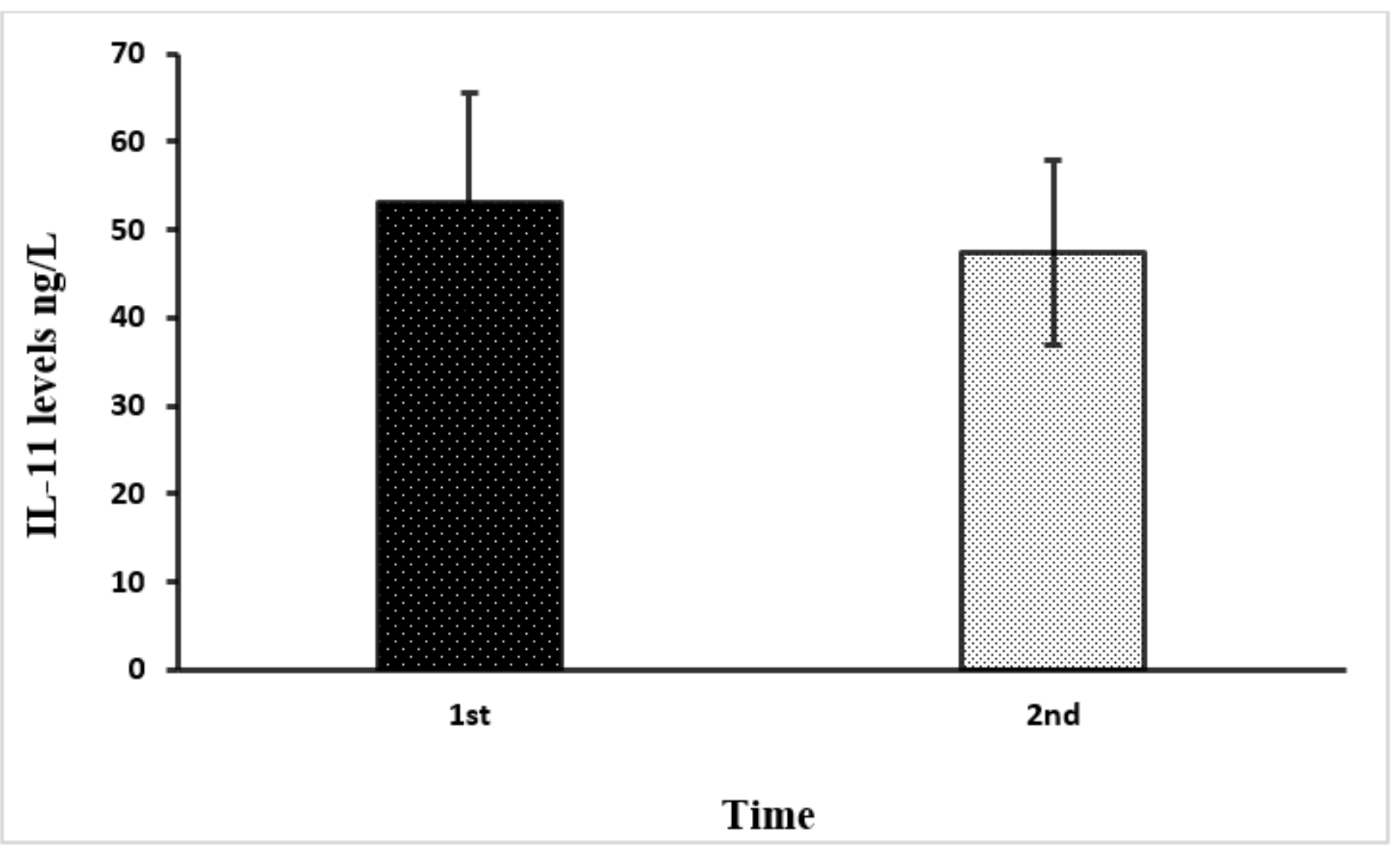

Figure 5

Serum IL-11 (ng/L) concentrations in patients with myocardial infarction. 1st time point: showed the first serum troponin measurements after the MI verification. 2nd time point: showed the second serum troponin measurements 72 hours after of Ml verification. Results are presented as mean $\pm S E M, n=40$. $P \leq 0.05^{\star}$. 